The NCCN Oncology Research Program (ORP) strives to improve the quality of life for patients and reduce cancer-related deaths by advancing cancer therapies through research. Since the program's establishment in 1999, the NCCN ORP has brought millions of dollars in research grants to investigators at NCCN Member Institutions. Research grants are provided to NCCN through collaborations with pharmaceutical and biotechnology companies; these grants are in turn used to support scientifically meritorious cancer research efforts.

NCCN ORP studies typically explore new avenues of clinical investigation and seek answers to important cancer-related questions. All studies are approved and funded through a scientific peer-review process and are overseen by the ORP.

This feature highlights an NCCN study funded through the grant mechanism.

doi: $10.6004 /$ jnccn.2021.0056

For more information on specific trials, including patient selection criteria, use the contact information listed with each study.

For more information on the NCCN ORP, including a complete detailing of the clinical studies currently underway at NCCN Member Institutions, go to www. nccn.org/education-research/nccn-oncologyresearch-program/clinical-trials.

\section{Use of Trifluridine/Tipiracil and Oxaliplatin as Induction Chemotherapy in Resectable Esophageal and Gastroesophageal Junction Adenocarcinoma}

Principal Investigator: Sarbajit Mukherjee, MD, MS

Condition: Esophageal or gastroesophageal junction adenocarcinoma

Institutions: Roswell Park Comprehensive Cancer Center, University of Chicago, University of Oklahoma/Stephenson Cancer Center

This is an open-label, multicenter, phase II study of trifluridine/tipiracil (FTD/TPI) administered with oxaliplatin before chemoradiation in patients with adenocarcinoma of the esophagus or gastroesophageal junction (GEJ).

Patients meeting inclusion criteria will receive 3 cycles of FTD/TPI (35 mg/m² twice daily) and oxaliplatin $\left(85 \mathrm{mg} / \mathrm{m}^{2}\right.$ ) before undergoing concurrent chemoradiation (standard-of-care radiation dose of 5,040 cGy will be used at all participating study sites) with carboplatin (area under the curve, 2) and paclitaxel $\left(50 \mathrm{mg} / \mathrm{m}^{2}\right)$ for 6 weeks, followed by surgery. FTD/TPI will be self-administered (in tablet form) twice daily by study participants and documented in the provided drug diary.

In this study, circulating tumor DNA (ctDNA) levels will be collected at 5 different time points: at diagnosis, after completion of induction chemotherapy, after completion of chemoradiation, after surgery, and at the time of disease recurrence, if this occurs. The aim is to correlate the ctDNA level with disease recurrence and metabolic response on $\mathrm{PET} / \mathrm{CT}$.

Accrual is expected to take 24 months $(\mathrm{N}=45)$ with an additional 12 months of follow-up on study. After completion of 1 year of on-study follow-up, patients will be followed (monitored for survival status) as per standard of care: year 1 through year 2 , every 3 to 6 months, every 6 to 12 months for 3 to 5 years, and then annually until disease progression or death, whichever occurs first. Estimated time frame for analysis of the primary endpoint is approximately 3 years after study commencement.

\section{Primary Objective:}

- Evaluate pathologic complete response rate in participants with esophageal or GEJ adenocarcinoma when FTD/TPI and oxaliplatin are used as induction chemotherapy prior to surgical resection

\section{Secondary Objectives:}

- Evaluate 2-year disease-free and overall survivals

- Determinate safety and tolerability of induction chemotherapy with FTD/TPI and oxaliplatin followed by standard chemoradiation and surgery

- Evaluate metabolic response to induction chemotherapy with FTD/TPI and oxaliplatin in participants with esophageal or GEJ adenocarcinoma prior to standard chemoradiation and surgical resection 


\section{Exploratory Objective:}

- Correlate ctDNA levels with disease recurrence and metabolic response on PET/CT

Contact: Sarbajit Mukherjee, MD, MS • 716-845-1300 •

Sarbajit.mukherjee@roswellpark.org

Coordinator: Sarah Chatley • Sarah.Chatley@roswellpark.org

ClinicalTrials.gov Identifier: NCT04097028 